\title{
REDUCED-COMPLEXITY I/Q TURBO DETECTOR FOR CONVOLUTIONAL-CODING ASSISTED SPACE-TIME TRELLIS CODING
}

\author{
B. L. Yeap, T. H. Liew and L. Hanzo \\ Dept. of Electr. and Comp. Sc., Univ. of Southampton, SO17 1BJ, UK. \\ Tel: +44-23-80593125, Fax: +44-23-80593045 \\ Email: 1h@ecs.soton.ac.uk \\ http://www-mobile.ecs.soton.ac.uk
}

\begin{abstract}
This paper presents a reduced-complexity turbo detector for convolutional-coding aided space-time trellis coded (STTC) schemes. The proposed technique is based on the separate in-phase/quadrature-phase $(\mathrm{I} / \mathrm{Q})$ detection principle developed for single-transmitter and single-receiver systems. Essentially, the reduced-complexity I/Q turbo detector (R-TD) decomposes the received signal into its constituent $I$ and $Q$ components and detects these components separately, hence reducing the number of possible signal combinations to be 'tested' by the detector. It is observed that the R-TD is capable of approaching the performance of the full-complexity turbo detector, while achieving a complexity reduction factor of 4.2 for a $R=\frac{1}{2}$ convolutional-coded 4-PSK 4-state STTC system communicating over two-path equal-weight Rayleigh fading channels. The performance of the convolutional-coded STTC schemes is also compared to that of the STTC benchmarker refraining from using channel coding, while maintaining a particular throughput. It was found that the convolutional-coded STTC system achieved a gain of $1.2 \mathrm{~dB}$ at $\mathrm{BER}=10^{-5}$, although at the expense of a significant increase in complexity.
\end{abstract}

\section{INTRODUCTION}

In the 3rd Generation (3G) mobile radio standard, spatial diversity has been proposed for increasing the link quality and therefore the capacity of the system. Spatial diversity can be implemented with the aid of multiple transmitters or receivers. However, for practical reasons it is more cost effective to employ the multiple antennas at the base station, rather than at the mobile station. Recently, transmit diversity techniques known as Space-time Trellis Coding (STTC) [1] have been advocated for providing additional diversity gains as well as for overcoming the limited capacity offered by the hostile fading wireless channels [3]. STTC relies on the joint design of channel coding, modulation, transmit diversity and the optional receiver diversity schemes. Typically, the detection of the STTC symbols consists of independent channel equalization and space-time trellis decoding operations.

Instead of implementing the channel equalization and STTC decoding operations independently, the performance of the STTC system can be further improved by performing both operations iteratively. This philosophy is based on that of turbo equalization [2, 3], which was first employed in a convolutional encoded BPSK system transmitting over dispersive channels. This technique performs channel equalization and channel decoding iteratively and has been shown to successfully mitigate the effects of channel ISI. Bauch et al. [4] adapted the turbo equalization technique for iterative channel equalization and STTC decoding. This approach yielded an improved performance due to the intelligent exploitation of the softdecision based feedback from the STTC decoder output to the equalizer's input.
Motivated by these trends, Reference [5, 3] improved the performance of the STTC system by employing additional channel encoding and turbo detection. However, due the high complexity of the turbo detector, investigations were limited to Channel Impulse Responses (CIR) associated with low dispersions. In this paper, we demonstrate, how the complexity of the turbo detector proposed in [5] can be reduced by employing the separate In-phase/Quadraturephase (I/Q) detection technique developed for single-transmitter and single-receiver systems [6]. Hence, investigations over channels exhibiting a high dispersion, such as a symbol-spaced five-path Rayleigh fading channel, were facilitated. In addition, we will also compare the performance of the $R=\frac{1}{2}$ channel coded 16-state 16QAM STTC scheme to that of the 4PSK STTC benchmarker refraining from using channel coding, while maintaining the same throughput. We commence our discourse by presenting an overview of the investigated system in Section 2. This is followed by an overview of the reduced complexity principles and a complexity analysis in Sections 3 and 4, respectively. Section 5 presents our simulation results and finally, we conclude in Section 6.

\section{SYSTEM OVERVIEW}

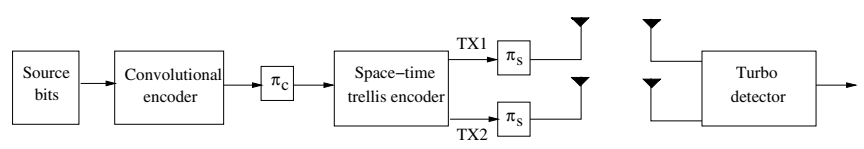

Figure 1: Schematic of the space-time trellis coded system employing turbo detection at the receiver. The notation $\pi_{s}$ represents the space-time trellis code's interleaver.

In the system model illustrated in Figure 1, we observe that the source bits are passed to a channel encoder, which subsequently directs the interleaved encoded bits to a two-transmitter STTC encoder, represented using the notation $\operatorname{STTC}(\mathrm{M}, n)$, where $\mathrm{M}$ denotes the modulation mode used and $n$ the number of states of the STTC encoder. Two different channel encoders were employed, namely a $\frac{1}{2}$-rate convolutional code having a constraint length of $K=5$ and octal generator polynomials of $G_{0}=35$ and $G_{1}=23$ as well as a $\frac{1}{2}$-rate, $K=7$ convolutional code using $G_{0}=133$ and $G_{1}=171$ [7] octal generator polynomials. At the output of the STTC encoder, the encoded symbols are interleaved by a random STTC interleaver represented as $\pi_{s}$ in Figure 1. Note that random interleavers are utilized for both the channel interleaver and for the STTC interleaver. Furthermore, the same interleaving rule is used for all transmit antennas in order to preserve the rank property of the space-time codes [1].

A transmission burst consisting of 144 data symbols was employed in our investigations. Additionally, two-path and five-path 
Rayleigh fading channels having equal symbol-spaced tap weights were used. The Rayleigh fading statistics obeyed a normalised Doppler frequency of $3.3615 \times 10^{-5}$, where the fading magnitude and phase was kept constant for the duration of a transmission burst. Furthermore, in order to characterize the best possible performance of these systems, the CIR was perfectly estimated at the receiver. As shown in Figure $1, R_{x}=2$ receive antennas were employed at the receiver. Again, turbo detection was employed, whereby the channel equalization, space-time trellis decoding and channel decoding operations were performed iteratively. The received signal at the $i$ th receiver can be written as:

$$
\begin{aligned}
r^{i}(t) & =\sum_{k=1}^{T_{x}=2} s^{k}(t) * h^{k i}(t)+n^{i}(t) \quad \mathrm{i}=1 \ldots R_{x}=2, \\
& =r_{I}^{i}(t)+j r_{Q}^{i}(t)
\end{aligned}
$$

where

$$
\begin{aligned}
r_{I}^{i}(t) & =\sum_{k=1}^{T_{x}=2}\left(s_{I}^{k}(t) * h_{I}^{k i}(t)-s_{Q}^{k}(t) * h_{Q}^{k i}(t)\right)+n_{I}^{i}(t) \\
j r_{Q}^{i}(t) & =\sum_{k=1}^{T_{x}=2} j\left(s_{Q}^{k}(t) * h_{I}^{k i}(t)+s_{I}^{k}(t) * h_{Q}^{k i}(t)\right)+j n_{Q}^{i}(t),
\end{aligned}
$$

and $s^{k}(t), r^{i}(t)$ and $n^{i}(t)$ denote the symbol transmitted from the $k$ th transmitter, the received signal of the $i$ th receive antenna and the AWGN imposed on the signal received by the $i$ th receive antenna, respectively. The notation $h^{k i}(t)$ refers to the CIR corresponding to the $k$ th transmit and $i$ th receive link, whereas $*$ represents convolution. Additionally, the subscripts $I$ and $Q$ represent the in-phase and quadrature-phase components, respectively.

\section{REDUCED COMPLEXITY TURBO DETECTION PRINCIPLES}

Recall that in Equation 2 the received I/Q signals, namely $r_{I}^{i}(t)$ and $r_{Q}^{i}(t)$, were shown to become dependent on $s_{I}^{k}(t)$ and $s_{Q}^{k}(t)$ after transmission over a complex channel. The inter-dependency between $s_{I}^{k}(t)$ and $s_{Q}^{k}(t)$ in the received quadrature signals $r_{I}^{i}(t)$ and $r_{Q}^{i}(t)$ is referred to here as cross-coupling. As a consequence of the cross-coupling the receiver has to consider an increased number of signal combinations for identifying the most likely transmitted symbol, hence necessitating a high number of equalizer trellis states. However, the number of trellis states to be considered can be reduced significantly, when the cross-coupling is removed such that the quadrature components of the decoupled channel output $r^{i^{\prime}}(t)$, which consists of $r_{I}^{i^{\prime}}(t)$ and $r_{Q}^{i^{\prime}}(t)$, are only dependent on $s_{I}^{k}(t)$ or $s_{Q}^{k}(t)$. This is the basic principle behind the reduced-complexity turbo detection scheme.

In order to perform the decoupling, the quadrature components of the signal estimates, namely $\hat{s}_{I}^{k}(t)$ and $\hat{s}_{Q}^{k}(t)$ of all transmitters, must first be generated. These quadrature signal estimates are obtained with the aid of [3]:

$$
\begin{gathered}
E\left\{s_{I}^{k}(t)\right\}=\hat{s}_{I}^{k}(t)=\sum_{u=0}^{\sqrt{\mathrm{M}}-1} s_{I ; u}^{k} \cdot P\left[s_{I ; u}^{k} \mid \hat{s}^{k}(t)\right] \\
E\left\{s_{Q}^{k}(t)\right\}=\hat{s}_{Q}^{k}(t)=\sum_{u=0}^{\sqrt{\mathrm{M}}-1} s_{Q ; u}^{k} \cdot P\left[s_{Q ; u}^{k} \mid \hat{s}^{k}(t)\right],
\end{gathered}
$$

where $E\{\cdot\}$ denotes the expectation or averaging operation, $\sqrt{M}$ is the number of constellation points in each quadrature arm of a particular modulation scheme and $\hat{s}^{k}(t)$ is the channel equalizer's sym- bol estimate of transmitter $k$. Subsequently, these estimated symbols are convolved with the in-phase and quadrature-phase CIR estimates of the $k$ th transmit and $i$ th receive link, in order to generate $\hat{s}_{I}^{k}(t) * \hat{h}_{I}^{k i}(t), j \hat{s}_{I}^{k}(t) * \hat{h}_{Q}^{k i}(t), \hat{s}_{Q}^{k}(t) * \hat{h}_{Q}^{k i}(t)$ and $j \hat{s}_{Q}^{k}(t) * \hat{h}_{I}^{k i}(t)$, where $k=1 \ldots T_{x}$ and $i=1 \ldots R_{x}$. With the aid of these signals the cross-coupling between the received quadrature signals can be removed using the procedures outlined in [3], such that we obtain the decoupled received signals $r_{I}^{i^{\prime}}(t)$ and $r_{Q}^{i^{\prime}}(t)$, which are only dependent on a particular quadrature component, namely on $s_{I}^{k}(t)$ or $s_{Q}^{k}(t)$, rather than on both. For example, the signal $r_{I}^{i^{\prime}}(t)$ can be generated by removing $\hat{s}_{Q}^{k}(t) * \hat{h}_{Q}^{k i}(t)$ and $j \hat{s}_{Q}^{k}(t) * \hat{h}_{I}^{k i}(t)$ from the received signal $r^{i}(t)$, yielding:

$$
\begin{aligned}
r_{I}^{i^{\prime}}(t)= & r^{i}(t)+\sum_{k=1}^{T_{x}=2}\left(\hat{s}_{Q}^{k}(t) * \hat{h}_{Q}^{k i}(t)-j \hat{s}_{Q}^{k}(t) * \hat{h}_{I}^{k i}(t)\right) \\
= & \sum_{k=1}^{T_{x}=2}\left(s_{I}^{k}(t) * h_{I}^{k i}(t)+j s_{I}^{k}(t) * h_{Q}^{k i}(t)+\right. \\
& \left.e\left(\hat{s}_{Q}^{k}(t), \hat{h}_{Q}^{k i}(t)\right)+j e\left(\hat{s}_{Q}^{k}(t), \hat{h}_{I}^{k i}(t)\right)\right)+n^{i}(t),
\end{aligned}
$$

where $e\left(\hat{s}_{Q}(t), \hat{h}_{Q}(t)\right)$ and $e\left(\hat{s}_{Q}(t), \hat{h}_{I}(t)\right)$ are the error terms expressed as:

$$
\begin{aligned}
& e\left(\hat{s}_{Q}^{k}(t), \hat{h}_{Q}^{k i}(t)\right)=r_{I}^{i}(t)+\hat{s}_{Q}^{k}(t) * \hat{h}_{Q}^{k i}(t) \\
& e\left(\hat{s}_{Q}^{k}(t), \hat{h}_{I}^{k i}(t)\right)=r_{Q}^{i}(t)-\hat{s}_{Q}^{k}(t) * \hat{h}_{I}^{k i}(t),
\end{aligned}
$$

which arise, when inaccurate CIR estimates and low-confidence symbol estimates are generated. Similarly, $r_{Q}^{i^{\prime}}(t)$ is obtained by subtracting $\hat{s}_{I}^{k}(t) * \hat{h}_{I}^{k i}(t)$ and $j \hat{s}_{I}^{k}(t) * \hat{h}_{Q}^{k i}(t)$, where $k=1 \ldots T_{x}$ and $i=1 \ldots R_{x}$ from $r^{i}(t)$. Although errors are introduced in the decoupling operation, it will be shown using the simulation results of Section 5, that the imperfect decoupling effects are compensated through successive turbo detection iterations and the performance approaches that of the turbo detector utilising the conventional fullcomplexity trellis-based equalizer [5].

Having highlighted the philosophy of the decoupling operation, we continue by describing the structure of the turbo detector, which incorporates the I/Q decoupling principle. In the first turbo detection iteration, a Multi-Variable Decision Feedback Equalizer (MVDFE) [8] is utilized. The MV-DFE is employed, since it constituted a low-complexity approach to providing initial estimates of the transmitted symbols, as compared to the more complex trellis-based equalizers. These soft decisions are passed to the STTC decoder, which subsequently generates the a posteriori information. The extrinsic information is extracted from the a posteriori information and directed to the channel decoder. Subsequently, the channel decoder computes the a posteriori information and extrinsic information. As shown in Equation 3, the a posteriori probability is utilized in the symbol regeneration process, which aids the decoupling operation, whereas the extrinsic information becomes the a priori information employed by the I/Q equalizer in the second turbo detection iteration. The decoupling is then performed using the regenerated symbols and with the aid of Equation 4. After the decoupling operation has been completed, the resultant signals corresponding to the inphase signal components $r_{I}^{i^{\prime}}(t)$ of all transmitters are directed to a I/Q equalizer, while the quadrature-phase signal components $r_{Q}^{i^{\prime}}(t)$ of all transmitters are processed by the second I/Q equalizer. These I/Q equalizers utilize Soft-In/Soft-Out (SISO) algorithms such as the Maximum A Posteriori (MAP) [9] algorithm, the Log-MAP algorithm $[10,11]$ or the Max-Log-MAP $[12,13]$, in order to compute the reliability information in the form of LLRs. In our investigations, we have utilized the Max-Log-MAP algorithm [12, 13] in both our I/Q equalizers and in the STTC decoder as well as in the convolutional decoder, since it constitutes a good compromise in terms of the achievable performance and the computational complexity imposed. 
The LLR values generated by the I/Q equalizers are deinterleaved by the STTC deinterleaver and passed to the STTC decoder. The STTC decoder computes the a posteriori LLRs, which are then processed for generating the extrinsic information. Subsequently, both the soft output information of the I/Q equalizers and the extrinsic information is employed by the channel decoder in order to generate the a posteriori LLR values. Before directing these LLR values back to the I/Q equalizer, the extrinsic LLR is once again extracted from the a posteriori LLRs computed by the channel decoder. As in the first iteration, the a posteriori LLR is used for symbol regeneration, while the extrinsic LLR values becomes the a priori LLR for the I/Q equalizers in the next turbo detection iteration. This iterative process is repeated, until the termination criterion stipulated is satisfied. In our investigations the iterations were curtailed, when no significant further performance gains could be obtained through additional iterations.

\section{COMPLEXITY ANALYSIS}

For simplicity, the complexity of the turbo detectors can be quantified in terms of the number of associated trellis transitions per information bit. Therefore, the complexity of the equalizer, which is dependent on the number of trellis transitions per coded bit, must be normalized by the number of information bits per symbol $T_{r}$, hence yielding the number of transitions computed per information bit.

For the conventional full-complexity trellis-based channel equalizer termed as CT-EQ, it can be shown that the complexity $\Lambda$ [CT-EQ] associated with equalizing STTC signals transmitted over a channel having complex weights and a delay spread of $\tau_{d}$ symbols is:

$$
\begin{aligned}
\Lambda \text { CT-EQ }] & =(\text { Num. of states } \cdot \text { Num. of transitions }) / T_{r} \\
& =\left(\mathrm{M}^{T_{x} \tau_{d}} \cdot \mathrm{M}^{T_{x}}\right) / T_{r}=\left(\mathrm{M}^{T_{x}\left(\tau_{d}+1\right)}\right) / T_{r},
\end{aligned}
$$

where again $\mathrm{M}, T_{x}$ and $T_{r}$ denote the number of constellation points, the number of transmitters and the number of information bits per symbol, respectively. By contrast, the complexity of a single I/Q equalizer $(\mathrm{I} / \mathrm{Q}-\mathrm{EQ})$ trellis stage is given by:

$$
\begin{aligned}
\Lambda[\mathrm{I} / \mathrm{Q}-\mathrm{EQ}] & =(\text { Num. of states } \cdot \text { Num. of transitions }) / T_{r} \\
& =\left(\sqrt{\mathrm{M}}^{T_{x}\left(\tau_{d}+1\right)}\right) / T_{r} .
\end{aligned}
$$

For the STTC(M,n) decoder, where $\mathrm{M}$ and $n$ denote the number of constellation points and the number of trellis states in the encoder, the complexity $\Lambda[\operatorname{STTC}(\mathrm{M}, n)]$ incurred is:

$$
\Lambda[\operatorname{STTC}(\mathrm{M}, n)]=(n \cdot \mathrm{M}) / T_{r} .
$$

Finally, the complexity of the $R=\frac{1}{2}$ and constraint length $K$ channel decoder $\Lambda[\mathrm{CC}]$ can be written as:

$$
\Lambda[\mathrm{CC}]=2^{K} \text {. }
$$

Having determined the complexity of the channel equalizer, that of the STTC decoder and that of the convolutional channel decoder as a function of the number of trellis transitions per information bit, the total complexity of the full-complexity turbo detector denoted by F-TD [5] can be estimated as:

$$
\Lambda[\mathrm{F}-\mathrm{TD}]=\left(\frac{\left(\left(\mathrm{M}^{T_{x}\left(\tau_{d}+1\right)}\right)+n \cdot \mathrm{M}\right)}{T_{r}}+2^{K}\right) \cdot \operatorname{Itr}[\mathrm{F}-\mathrm{TD}],
$$

while the complexity of R-TD as:

$$
\begin{gathered}
\Lambda[\mathrm{R}-\mathrm{TD}]=\left(\frac{n \cdot \mathrm{M}}{T_{r}}+2^{K}\right)+ \\
\left(\frac{2 \cdot \sqrt{\mathrm{M}}^{T_{x}\left(\tau_{d}+1\right)}+n \cdot \mathrm{M}}{T_{r}}+2^{K}\right) \times(\mathrm{Itr}[\mathrm{R}-\mathrm{TD}]-1)
\end{gathered}
$$

where $\operatorname{Itr}[$ ] denotes the number of turbo detection iterations. A factor of two was introduced in the term $2 \cdot \sqrt{\mathrm{M}}^{T_{x}\left(\tau_{d}+1\right)}$ found in Equation 11, since two I/Q-EQs are required for performing the equalization. In Equation 11, the terms within the first brackets on the right hand side represent the complexity incurred in the first R-TD iteration, where the combination of a MV-DFE, STTC decoder and a convolutional decoder was employed. For the sake of simplicity we have assumed that the complexity of the MV-DFE is negligible, when compared to the complexity of the I/Q-EQ and CT-EQ. Therefore, the complexity of the R-TD in the first iteration is only dependent on the complexity of the STTC decoder and that of the convolutional decoder. The remaining terms within the second brackets correspond to the complexity of the subsequent R-TD iterations.

\section{RESULTS AND DISCUSSION}

In this section, we compare the performance of the F-TD proposed in [5] to that of the R-TD described in this contribution. These turbo detectors are utilized in conjunction with the $R=\frac{1}{2}$ and constraint length $K=5$ convolutional-coded 4-state QPSK STTC employing a 9216-symbol STTC interleaver and a 18430-bit channel interleaver for transmission over a two-path, equal-weight and symbol-spaced Rayleigh fading channel having a normalised Doppler frequency of $3.3615 \times 10^{-5}$. In Figure 2 it was observed that after performing three turbo detection iterations the R-TD was capable of approaching the performance of the F-TD, which also utilized three turbo detection iterations. No further improvements were obtained by performing additional turbo detection iterations. Using Equations 10 and 11 , we note that complexity of the R-TD is a factor of 4.2 lower than that of the F-TD scheme. Figure 3 shows the performance of the

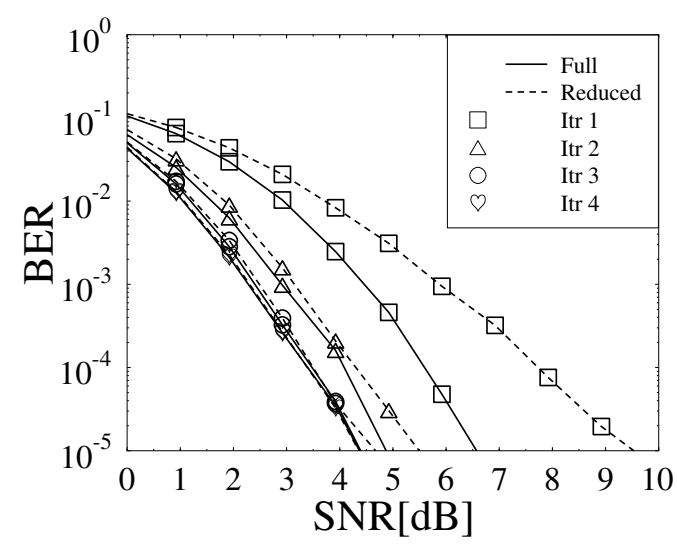

Figure 2: Performance comparison of the F-TD and R-TD in conjunction with the convolutional coded STTC $(4,4)$ scheme using a 9216-symbol STTC interleaver and a 18430bit channel interleaver for transmission over a two-path, equal-weight, and symbol-spaced Rayleigh fading CIR having a normalised Doppler frequency of $3.3615 \times 10^{-5}$.

R-TD scheme in conjunction with the STTC $(4,4)$ system communicating over a symbol-spaced five-path, equal tap-weight Rayleigh fading channel. For this CIR, the F-TD scheme cannot be realistically studied by simulation, since the complexity of the trellis-based equalizer alone is already associated with $10^{6}$ transitions per trellis stage. Despite experiencing long delay spreads, the R-TD was capable of detecting signals transmitted over such high-dispersion channels, while attaining a good BER performance. Assuming that the F-TD could have been simulated and two turbo detection itera- 


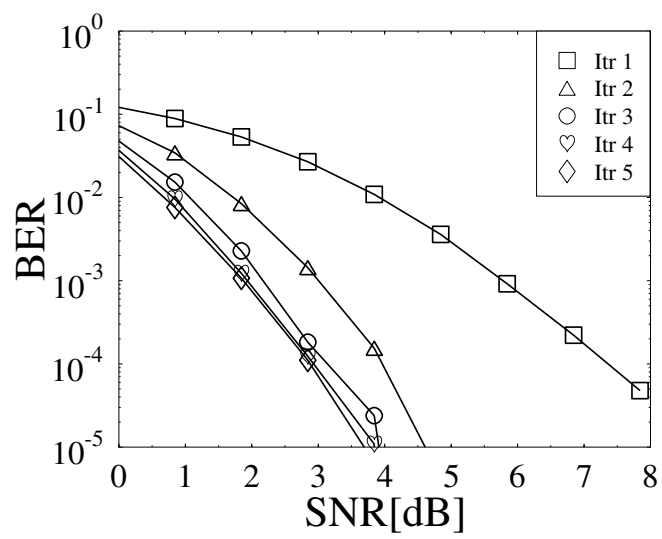

Figure 3: Performance of the R-TD in conjunction with the $R=\frac{1}{2}$ convolutional coded STTC $(4,4)$ scheme using a 9216symbol STTC interleaver and a 18430-bit channel interleaver for transmission over a five-path, equal-weight, and symbol-spaced Rayleigh fading CIR having a normalised Doppler frequency of $3.3615 \times 10^{-5}$.

tions were required, the R-TD would have achieved a complexity reduction of a factor of 331.0.

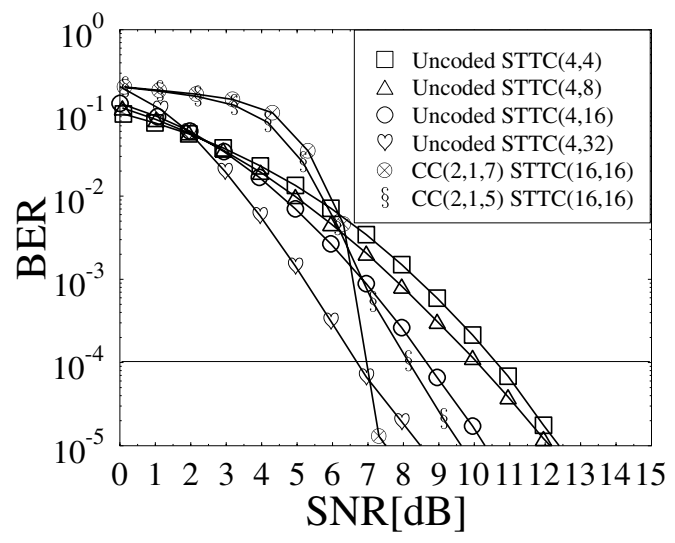

Figure 4: Comparing the R-TD performance of the $R=\frac{1}{2}$ convolutional coded $\operatorname{STTC}(16,16)$ scheme to that of F-TD STTC $(4, n)$ benchmarkers refraining from using channel coding, where $n=4,8,16$ and 32 states, when transmitting over a two-path, equal-weight, and symbol-spaced Rayleigh fading channel having a normalised Doppler frequency of $3.3615 \times 10^{-5}$.

Next, we investigated the performance of the channel-coded STTC scheme using R-TD to that of the STTC systems refraining from using channel coding but utilizing the full-complexity turbo detector [4], while maintaining the same overall throughput for all schemes. In order to ensure that the $R=\frac{1}{2}$ convolutional coded STTC scheme had the same throughput as the 'non-channel-coded' STTC $(4, n)$ systems, a higher-order modulation mode based STTC, such as the STTC $(16,16)$, was utilized in conjunction with the $R=\frac{1}{2}$ convolutional code. Specifically, we compared the F-TD performance of the 'non-channel-coded' $\operatorname{STTC}(4,4), \operatorname{STTC}(4,8), \operatorname{STTC}(4,16)$ and
STTC $(4,32)$ schemes to that of the $R=\frac{1}{2}$ convolutional-coded STTC $(16,16)$ scheme using the R-TD technique. In the channelcoded STTC $(16,16)$ scheme, two different constraint length convolutional codes were employed, namely the constraint length $K=$ 5 convolutional code and the $K=7$ convolutional code. Both schemes employed a 9216-symbol random STTC interleaver.

From Figure 4, it was observed that the $R=\frac{1}{2}$ and constraint length $K=5$ convolutional-coded STTC $(16,16)$ scheme employing R-TD and eight turbo detection iterations was capable of outperforming the 'non-channel-coded' STTC $(4,4)$, STTC $(4,8)$ and STTC$(4,16)$ schemes by $2.5 \mathrm{~dB}, 1.8 \mathrm{~dB}$ and $0.5 \mathrm{~dB}$, respectively at $\mathrm{BER}=$ $10^{-4}$. However, the 'non-channel-coded' STTC $(4,32)$ was observed to require $1.6 \mathrm{~dB}$ lower SNR than the convolutional-coded STTC$(16,16)$ scheme, in order to achieve the same BER. When the higher constraint length convolutional code was utilized, namely the $K=$ 7 convolutional code, the performance of the convolutional-coded STTC $(16,16)$ scheme approached that of the 'non-channel-coded' STTC $(4,32)$ system at $\mathrm{BER}=10^{-4}$, while achieving a gain of $1.2 \mathrm{~dB}$ at $\mathrm{BER}=10^{-5}$.

Figure 5 highlights the complexity required by both the channelcoded STTC $(16,16)$ system employing R-TD and that of the 'nonchannel-coded' STTC $(4, n)$ scheme utilizing the F-TD technique, in order to achieve a BER of $10^{-4}$. It was observed that the $R=\frac{1}{2}$ $K=7$ convolutional-coded $\operatorname{STTC}(16,16)$ system invoking eight turbo detection iterations outperforms the 'non-channel-coded' STTC$(4,4)$, STTC $(4,8)$ and $\operatorname{STTC}(4,16)$ schemes using two iterations, although at the cost of a factor of 14.1, 13.3 and 12.0, higher complexity, respectively. When compared to the 'non-channel-coded' STTC $(4,32)$ scheme using F-TD and performing three turbo detection iterations, the $R=\frac{1}{2}$ and constraint length $K=7$ convolutionalcoded STTC $(16,16)$ system using R-TD required a comparable SNR at $\mathrm{BER}=10^{-4}$, while achieving an SNR gain of $1.2 \mathrm{~dB}$ at $\mathrm{BER}=$ $10^{-5}$. This gain was achieved at the expense of 6.7 times higher computational complexity.

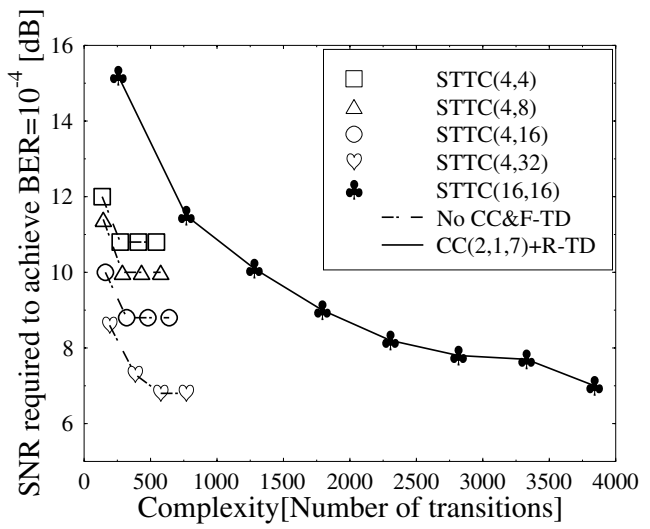

Figure 5: Comparing the complexity of F-TD assisted STTC $(4,4)$, STTC $(4,8), \operatorname{STTC}(4,16)$ and $\operatorname{STTC}(4,32)$ systems with that of the R-TD convolutional-coded $\operatorname{STTC}(16,16)$ scheme for transmission over a two-path, equal-weight, and symbol-spaced Rayleigh fading CIR having a normalised Doppler frequency of $3.3615 \times 10^{-5}$. In each curve, the complexity increases upon increasing the number of turbo detection iterations.

For typical speech and video systems the target BER is between $10^{-4}$ and $10^{-3}$. Therefore, for such schemes the turbo-detected STTC scheme refraining using channel coding is the more robust choice. Furthermore, the complexity incurred by these systems is 
significantly lower, than that of the channel-coded STTC schemes. From these results it can be concluded that for turbo-detected STTC schemes of the same throughput, it is better to invest the available complexity in implementing higher complexity STTC codes such as the STTC $(4,32)$ than in concatenating a powerful channel encoder with a weak STTC code, such as the STTC $(16,16)$.

\section{CONCLUSIONS}

In conclusion, it was observed in Figure 2 that the R-TD is capable of reducing the complexity of the STTC system, without significantly sacrificing the achievable performance. For channels having short delay spreads, such as the two-path, symbol-spaced and equal tap-weight channel, the R-TD scheme approached the performance of the F-TD arrangement, while achieving a complexity reduction factor of 4.2. For the five-path, symbol-spaced and equalweight Rayleigh fading channel, the F-TD scheme was not implemented, since the complexity of the trellis-based equalizer alone already corresponds to $10^{6}$ transitions per trellis stage. However, it was observed that the R-TD was capable of detecting signals transmitted over such channels, while attaining a good BER performance. Assuming that the F-TD could have been simulated and two critical turbo detection iterations were required, the R-TD would have achieved a complexity reduction factor of 331.0. When comparing STTC schemes of the same overall throughput, it was observed from Figure 4 that the turbo-detected 'non-channel-coded' $\operatorname{STTC}(4,32)$ system required a similar SNR to that of the $R=\frac{1}{2}$ and constraint length $K=7$ convolutional-coded $\operatorname{STTC}(16,16)$ scheme, in order to achieve the BER of $10^{-4}$. The convolutional-coded STTC $(16,16)$ only outperformed the 'non-channel-coded' $\operatorname{STTC}(4,32)$ arrangement for SNRs in excess of $7 \mathrm{~dB}$, but at the expense of 6.7 times higher computational complexity.

\section{Acknowledgement}

This work has been funded in the framework of the IST project SCOUT, which is partly funded by the European Union. The authors would like to acknowledge the contributions of their colleagues.

\section{REFERENCES}

[1] V. Tarokh, N. Seshadri, and A. R. Calderbank, "Space-Time Codes for High Data Rate Wireless Communication: Performance Criterion and Code Construction," IEEE Transactions on Information Theory, vol. 44, pp. 744-765, March 1998.

[2] C. Douillard, A. Picart, M. Jézéquel, P. Didier, C. Berrou, and A. Glavieux, "Iterative correction of intersymbol interference: Turbo-equalization," European Transactions on Communications, vol. 6, pp. 507-511, September-October 1995.

[3] L. Hanzo, T. H. Liew, and B. L. Yeap, Turbo Coding, Turbo Equalisation and Space-Time Coding for Transmission over Fading Channels. John-Wiley IEEE Press, 2002.

[4] G. Bauch, A. F. Naguib, and N. Seshadri, "MAP Equalization of Space-time Coded Signals over Frequency Selective Channels," in Proceedings of the Wireless Communications and Networking Conference, (New Orleans, USA), September 1999.

[5] B. L. Yeap and T. H. Liew and L. Hanzo, "Turbo Equalization of Serially Concatenated Systematic Convolutional Codes and Systematic Space Time Trellis Codes," IEEE Vehicular Technology Conference, p. 119 (CDROM), May 2001.

[6] B. L. Yeap, C. H. Wong, and L. Hanzo, "Reduced complexity in-phase/quadrature-phase turbo equalisation with iterative channel estimation," in IEEE International Conference on Communications 2001, (Helsinki, Finland), pp. 422-426, 1115 June 2001.
[7] "Digital Video Broadcast (DVB): Framing structure, channel coding and modulation for 11/12 GHz Satellite Services," pp. 12-14, August 1997.

[8] C. Tidestav, M. Sternad, and A. Ahlén, "Reuse Within a Cell - Interference Rejection or Multiuser Detection," IEEE Transactions on Communications, vol. 47, pp. 1511-1522, October 1999.

[9] L. R. Bahl, J. Cocke, F. Jelinek, and J. Raviv, "Optimal Decoding of Linear Codes for Minimising Symbol Error Rate," IEEE Transactions on Information Theory, pp. 284-287, March 1974.

[10] P. Robertson, E. Villebrun, and P. Hoeher, "A Comparison of Optimal and Sub-Optimal MAP Decoding Algorithms Operating in the Log Domain," in Proceedings of the International Conference on Communications, (Seattle, United States), pp. 1009-1013, 18-22 June 1995.

[11] P. Robertson, P. Hoeher, and E. Villebrun, "Optimal and suboptimal maximum a posteriori algorithms suitable for turbo decoding," European Transactions on Telecommunications, vol. 8, pp. 119-125, March/April 1997.

[12] W. Koch and A. Baier, "Optimum and Sub-Optimum Detection of Coded Data Disturbed by Time-Varying Inter-Symbol Interference," in Proceedings of the IEEE Global Telecommunications Conference 1990, (San Diego, United States), pp. 16791684, 2-5 December 1990.

[13] J. A. Erfanian, S. Pasupathy, and G. Gulak, "Reduced Complexity Symbol Detectors with Parallel Structures for ISI Channels," IEEE Transactions on Communications, vol. 42, pp. 1661-1671, February/March/April 1994. 